\title{
Enhancement of spatiotemporal regularity in an optimal window of random coupling
}

\author{
Swarup Poria * \\ Department of Mathematics, Midnapore College, \\ Midnapore, 721 101, West Bengal, India \\ Manish Dev Shrimali ${ }^{\dagger}$ \\ Department of Physics, Dayanand College, Ajmer, 305 001, India \\ Sudeshna Sinha \\ The Institute of Mathematical Sciences, \\ Taramani, Chennai 600 113, India
}

\begin{abstract}
We investigate the spatiotemporal dynamics of a lattice of coupled chaotic maps whose coupling connections are dynamically rewired to random sites with probability $p$, namely at any instance of time, with probability $p$ a regular link is switched to a random one. In a range of weak coupling, where spatiotemporal chaos exists for regular lattices (i.e. for $p=0$ ), we find that $p>0$ yields synchronized periodic orbits. Further we observe that this regularity occurs over a window of $p$ values, beyond which the basin of attraction of the synchronized cycle shrinks to zero. Thus we have evidence of an optimal range of randomness in coupling connections, where spatiotemporal regularity is efficiently obtained. This is in contrast to the commonly observed monotonic increase of synchronization with increasing $p$, as seen for instance, in the strong coupling regime of the very same system.
\end{abstract}

\footnotetext{
* e-mail: swarup_p@yahoo.com

$\dagger$ e-mail: m.shrimali@gmail.com

$\ddagger$ e-mail: sudeshna@imsc.res.in
} 


\section{INTRODUCTION}

The dynamics of spatially extended systems has been a focus of intense research activity in the past two decades. In recent years it has become evident that modelling large interactive systems by finite dimensional lattices on one hand, and fully random networks on the other, is inadequate, as various networks, ranging from collaborations of scientists to metabolic networks, do not to fit in either paradigm [1]. Some alternate scenarios have been suggested, such as the small-world network [2]. Here one starts with a regular structure on a lattice, for instance nearest neighbour interactions. Then each regular link from a site is rewired randomly with probability $p$. This model is proposed to mimic real life situations in which non-local connections exist along with predominantly local connections.

There is much evidence that random nonlocal connections, even in a small fraction, significantly affect geometrical properties, like characteristic path length [3]. However its implications for dynamical properties is still unclear and even conflicting. While the dynamics of coupled oscillators and coupled maps on regular lattices has been extensively investigated [4], there have been far fewer studies on the sptiotemporal features of nonlinear elements on more general network topologies [ㅍ, 6].

Most existing case studies of coupled networks of dynamical elements indicate that features, such as degree of synchronization, vary monotonically with $p$. That is, it is observed that most dynamical properties interpolate between the limits of regular and random connections without in any sense being "optimal" or more pronounced at some intermediate value of $p$.

In this paper however we will provide evidence of a system where there exists a window of randomness where one obtains special dynamical features which cannot be anticipated from a simple interpolation between the regular and random limits. In particular we will show the pronounced enhancement of spatiotemporal order in the system, in am intermediate window of rewiring probability $p$. Our observations are markedly distinct from the commonly observed monotonic dependence of synchronization properties on $p$, as seen for instance, in the strong coupling regime of the very same system [6]. 


\section{MODEL}

Specifically we consider a one-dimensional ring of coupled logistic maps. The sites are denoted by integers $i=1, \ldots, N$, where $N$ is the linear size of the lattice. On each site is defined a continuous state variable denoted by $x_{n}(i)$, which corresponds to the physical variable of interest. The evolution of this lattice, under standard interactions with the nearest neighbours on either side, in discrete time $n$, is given by

$$
x_{n+1}(i)=(1-\epsilon) f\left(x_{n}(i)\right)+\frac{\epsilon}{2}\left\{x_{n}(i+1)+x_{n}(i-1)\right\}
$$

The strength of coupling is given by $\epsilon$. The local on-site map is chosen to be the fully chaotic logistic map: $f(x)=4 x(1-x)$, as this map has widespread relevance as a prototype of low dimensional chaos.

Now we will consider the above system with its coupling connections rewired randomly in varying degrees, and try to determine what dynamical properties are significantly affected by the way connections are made between elements. In our study, at every update we will connect a site with probability $p$ to randomly chosen sites, and with probability $(1-p)$ to nearest neighbours, as in Eqn. 1. That is, at every instant a fraction $p$ of randomly chosen nearest neighbour links are replaced by random links. The case of $p=0$ corresponds to the usual nearest neighbour interaction, while $p=1$, corresponds to completely random

coupling. Note that the random connections are dynamic here, as the random links are switched around at every update. This is in contrast to most studies which consider static (quenched) random connections.

\section{EMERGENCE OF SYNCRHONIZED CYCLES FROM SPATIOTEMPORAL CHAOS}

We will now present numerical evidence that random rewiring has a pronounced effect on spatiotemporal order. The numerical results here have been obtained by sampling a large set of random initial conditions $\left(\sim 10^{4}\right)$, and with lattice sizes ranging from 50 to 1000 .

Fig. 1 displays the spatiotemporal state of the network, $x_{n}(i), i=1, \ldots N$, with respect to coupling strength $\epsilon$, for the case of regular nearest neighbour interactions (i.e. $p=0$ ) and for the case of random coupling with probability $p=0.19$ and $p=0.6$. It is clearly seen that the standard nearest neighbour coupling does not yield regularity anywhere in 
the entire coupling range, while randomly rewiring with small probability $p=0.15$ creates a window in parameter space where synchronized cycles gains stability. Note that different periodcities of the synchronized cycles are obatined under different coupling strengths, in the window of synchronization. Interestingly however, when $p$ is large this window of complete spatiotemporal regularity is lost again (see Fig. 1c).

In order to quantify this phenomena, we find the fraction of random initial conditions that get synchronized (after long transience) This provides a measure of the size of the basin of attraction of the synchronized state, denoted by $B$ (see Fig. 2). When $B=1$ we obtain a global attractor for the synchronized cycles.

Fig. 3 shows the synchronized basin size $B$ with respect to rewiring probability $p$, for different coupling strengths. It is clearly evident from the figure that for the regular lattice limit of $p=0$, the synchronized basin size is close to zero. As $p>0$ the basin for the synchronized state increases rapidly. For instance, for $p=0.01$ in Fig. 3, the synchronized state is the global attractor of the dynamics, i.e. $B=1$. However, as $p$ exceeds an optimal window, the basin size decreases rapidly, becoming zero again for high $p$. Thus there exists a range of $p$ values where synchronized cycles gain stability. This observation is quantified in Fig. 4 which gives the range of rewiring probability, $R$, over which $B=1$ for different coupling strengths.

It is evident then that there is a non-monotonic enhancement of synchronization with increasing randomness in coupling connections, and synchronization is most enhanced in some window of $p$ values. This is in sharp contrast to the monotonic increase in synchronization with increasing randomness in connectivity, in the strong coupling regime in the same system.

\section{EFFICIENCY OF SYNCHRONIZATION}

We calculate the average time taken for systems with random initial conditions to synchronize, and denote this as $\langle T\rangle$. This quantity, which is a measure of the efficiency of synchronization, is displyed in Figs. 5-6.

It is evident from Fig. 5 that $\langle T\rangle$ first decreases slightly as $p$ increases and then very sharply rises, by orders of magnitude, especially for larger lattices. It is clear then that synchronization is most efficient in some range of low $p$ values. So in order to obtain fast 


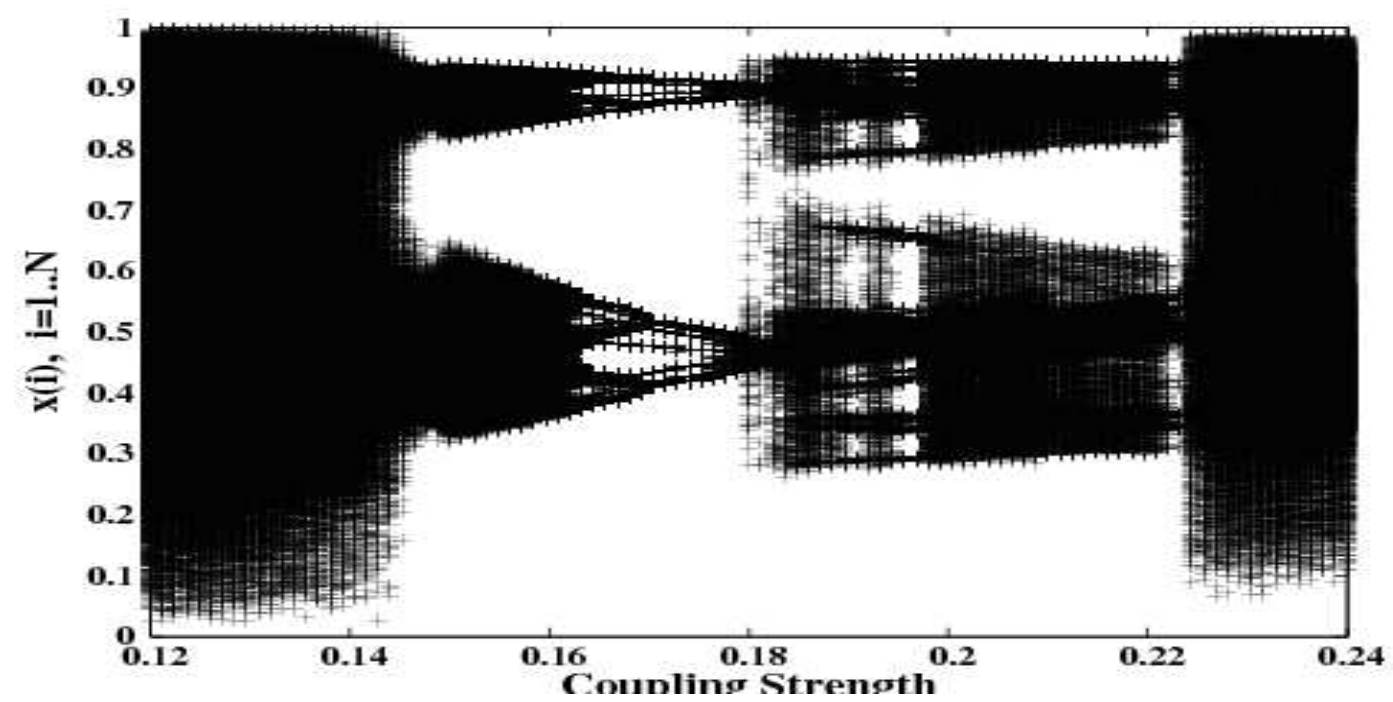

(a)

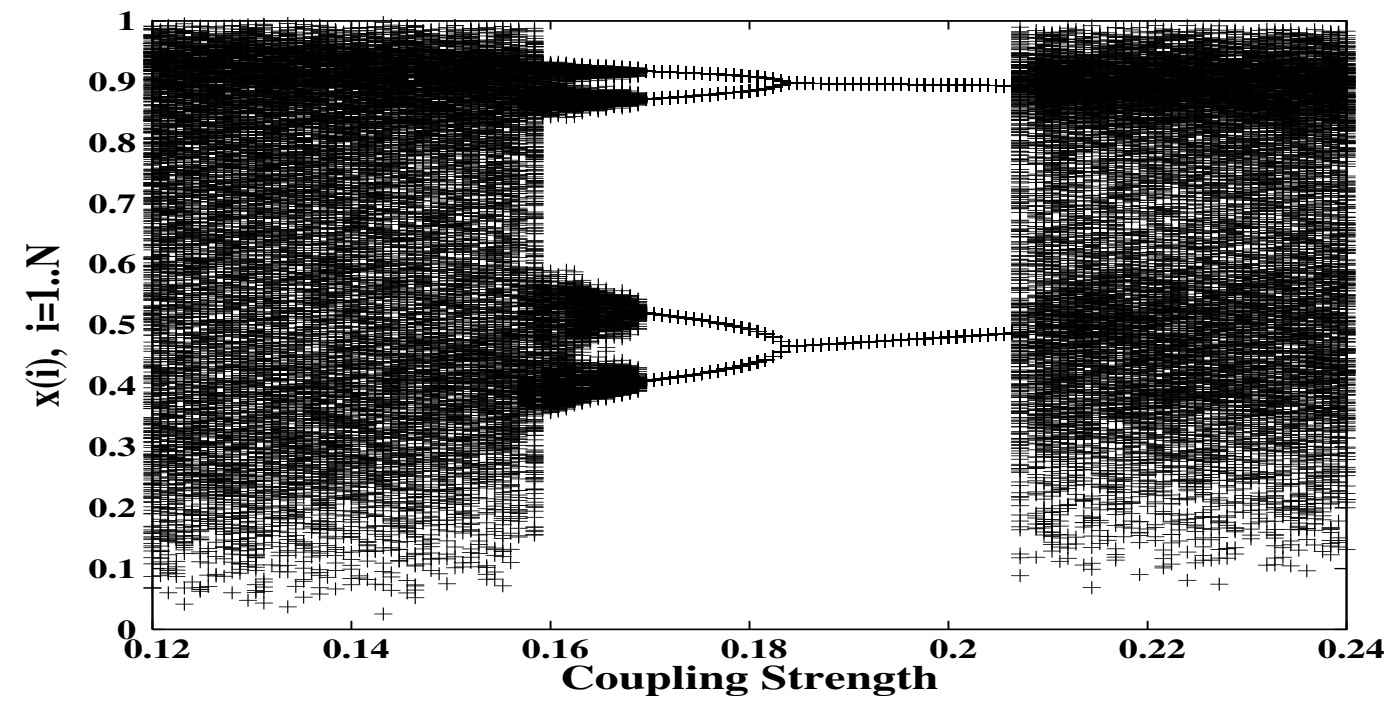

(b)

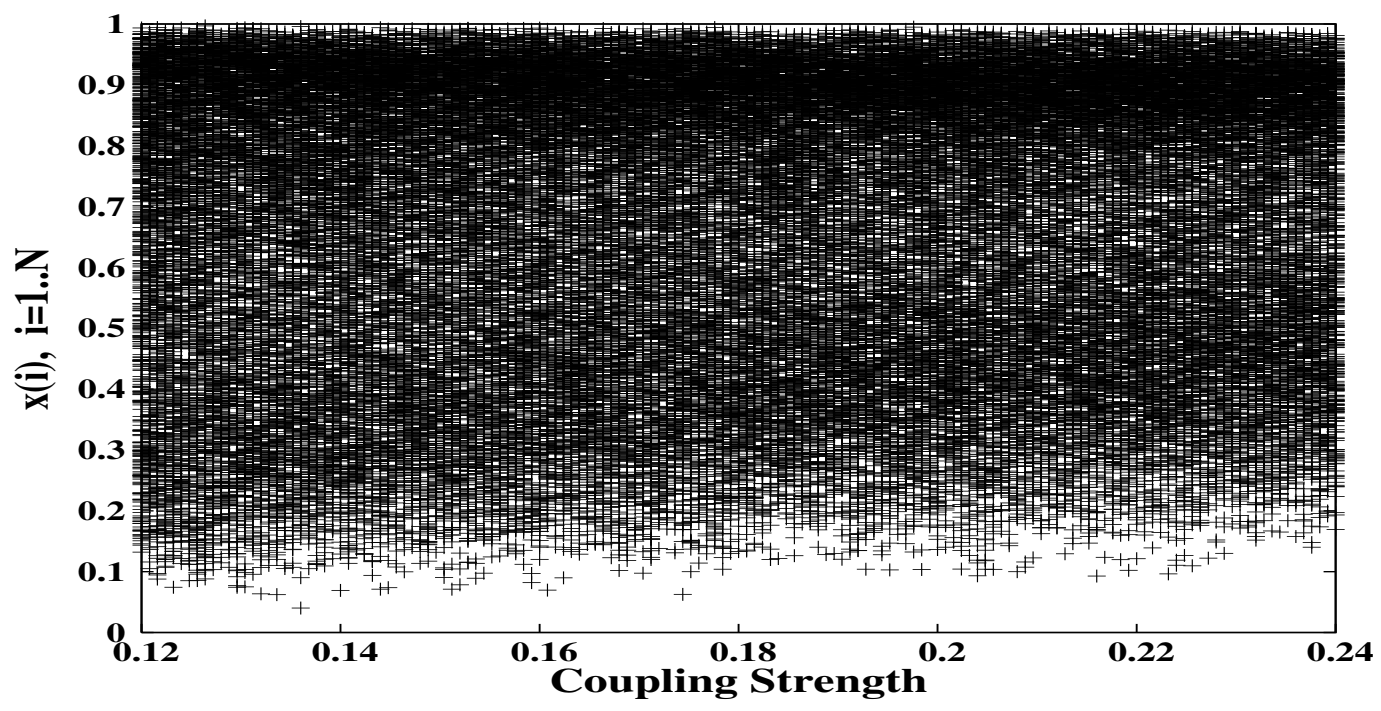

(c)

FIG. 1: Bifurcation diagram showing the state of the lattice $x_{n}(i), i=1, \ldots N(N=50)$, over $n=1, \ldots 25$, with respect to coupling strength $\epsilon$, for (a) $p=0$, (b) $p=0.19$ and (c) $p=0.6$. The synchronized 2 and 4 cycles are clearly evident gुn (b), but not in (a) or (c). 


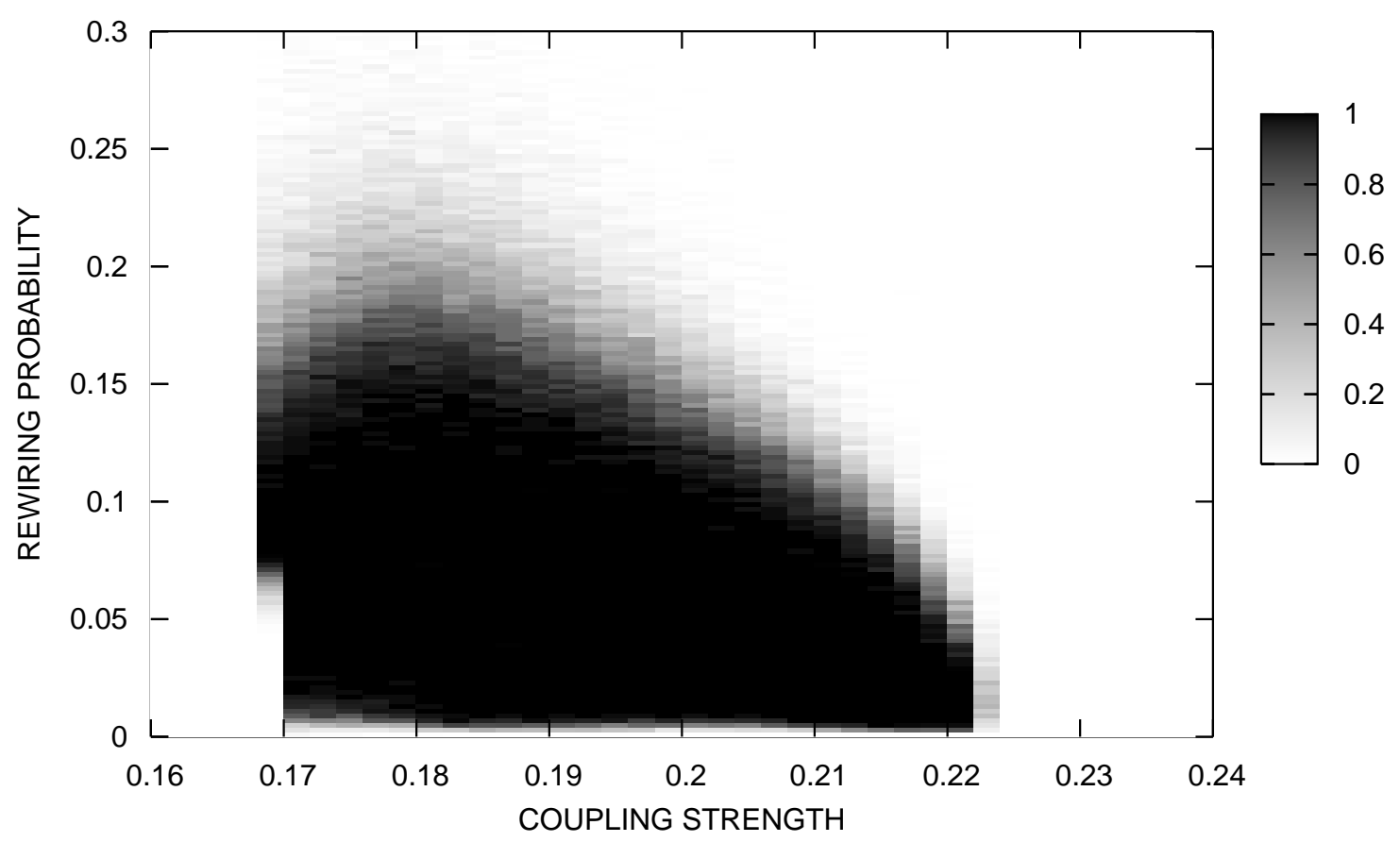

FIG. 2: Size of the basin of attraction of the synchronized cycles as a density plot in the space of coupling strength $\epsilon$ and random rewiring probability $p$, for a system of size 100. Here transience is 10000.

and reliable synchronization it is a good strategy to choose low $p$, as this ensures a global attractor for the synchronized cycles, as well as a fast approach to the synchronized state from a random initial state.

As a consequence of the above, the size of the basin of attraction for the synchronized cycles, $B$, depends on the allowed transience. Fig. 6 shows the increase in the basin $B$ as transience is increased. However note that this increase is very slow, and increasing transience by 2 orders of magnitude changes the global attractor width in $p$ space by a small amount.

Caveat: it appears possible, from the above observations, that in the limit of $T \rightarrow \infty$ a synchronized state may be reached for larger lattices as well. But it should be underscored that transience is always finite, and it is indeed very pertinent to know what generically 


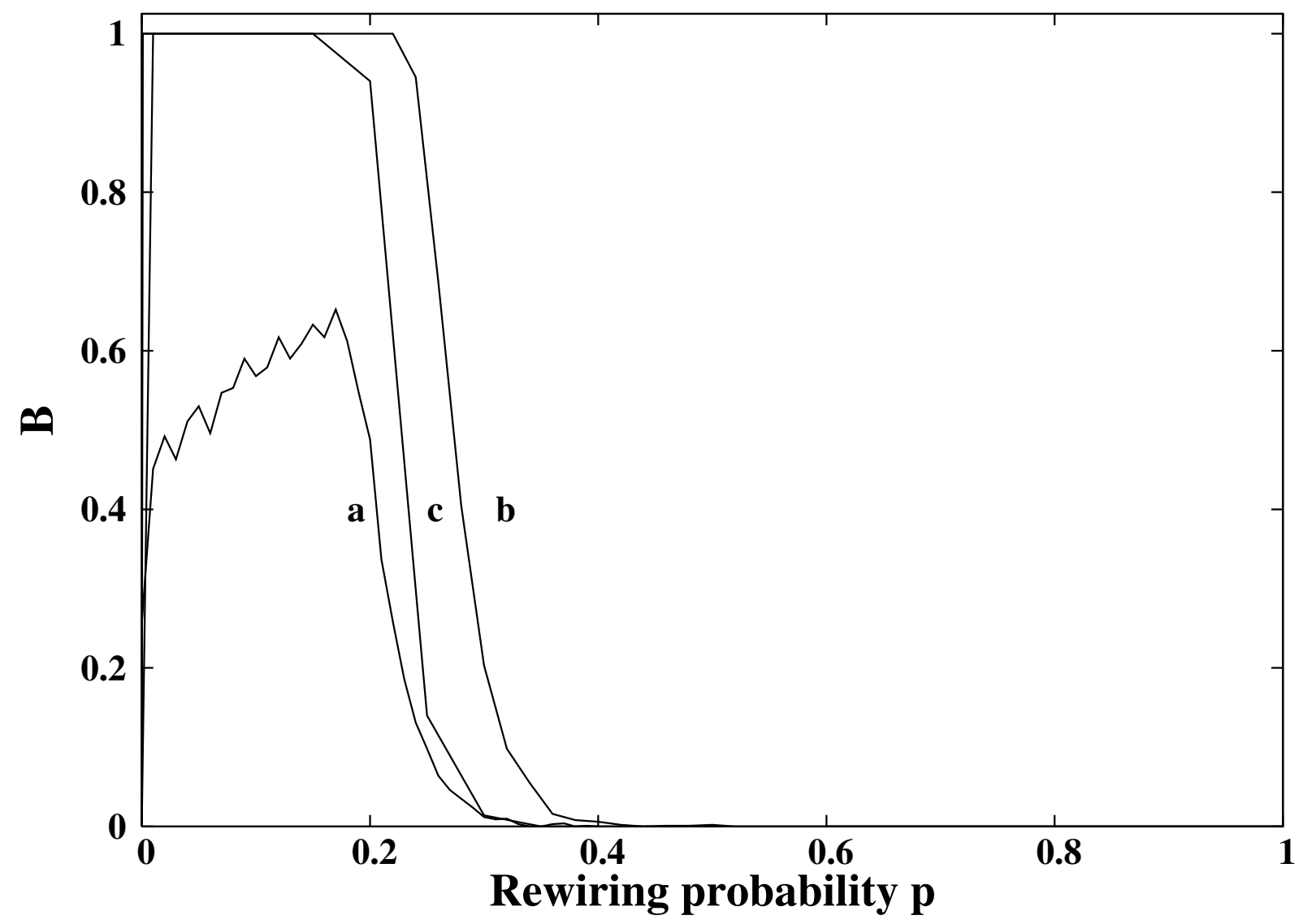

FIG. 3: Size of the basin of attraction of the synchronized state vs rewiring probability $p$ for a system of size 100 for coupling strengths (a) $\epsilon=0.16$, (b) $\epsilon=0.19$ (c) $\epsilon=0.20$. Here transience is $5 \times 10^{5}$.

happens in the limit of finite, but large $\left(\sim O\left(N^{2}\right)\right)$, transience, as is the case for the studies in this paper.

\section{CONCLUSIONS}

We have investigated the spatiotemporal dynamics of a network of coupled chaotic logistic maps, with varying degrees of randomness in coupling connections. We find a window in coupling parameter space, in the weak coupling regime, where random rewiring induces spatiotemporal order. Interestingly the basin of attraction for the synchronized cycles varies non-monotonically with rewiring probability $p$. As $p$ is increased, the basin of attraction of the synchronized state rapidly increases. At an intermediate (small) value of $p$ the synchro- 


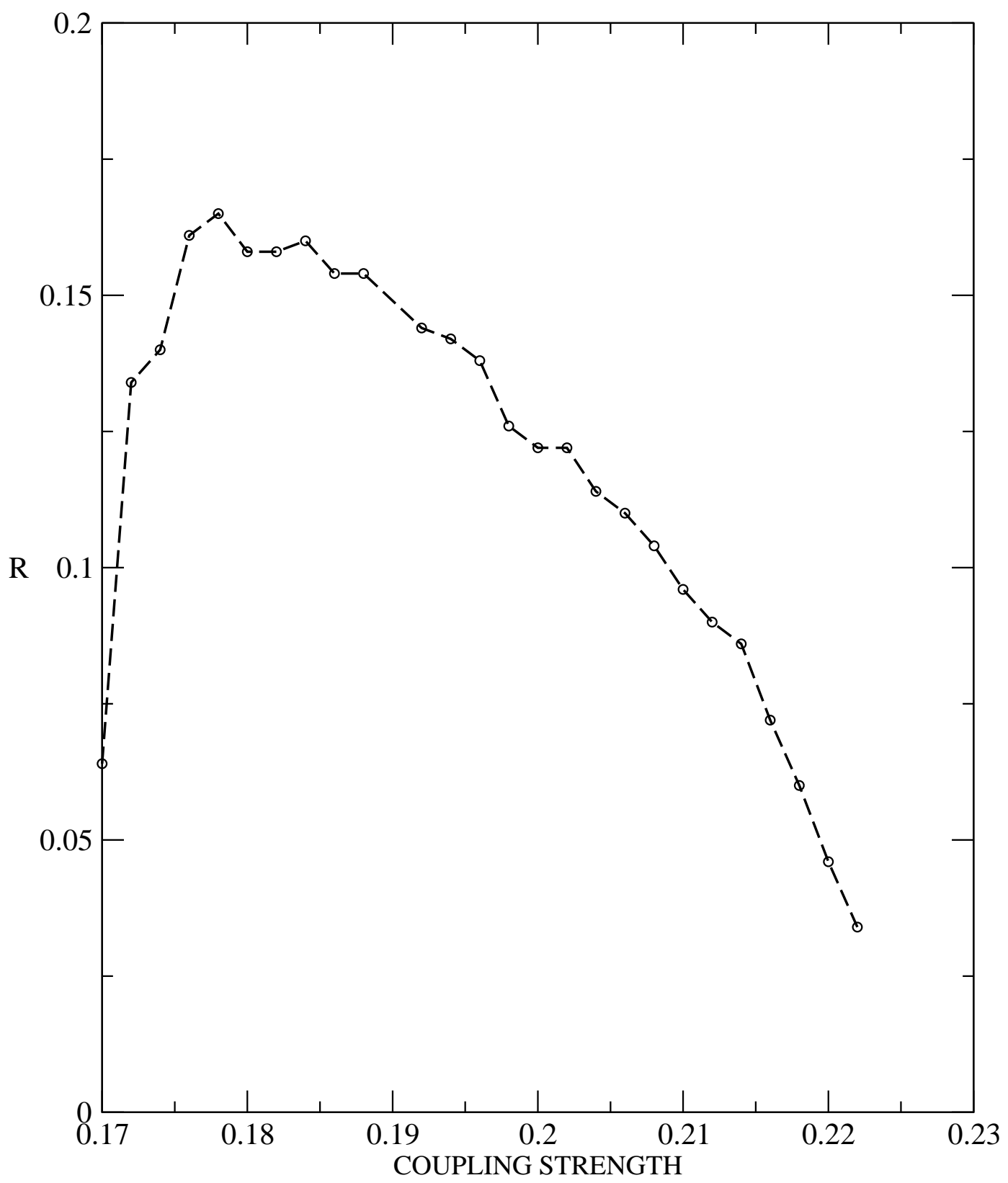

FIG. 4: Range of rewiring probability $R$, over which synchronization is achieved vs. coupling strength $\epsilon$. Here system size is 100 and transience is 10000 .

nized state becomes the global attractor of the system with (almost) all initial conditions being attracted to the synchronized state. However, interestingly, as $p$ is further increased, the basin of attraction of the synchronized state shrinks to zero again. Thus we have strong evidence of the pronounced enhancement of spatiotemporal order in an intermediate window of $p$. This is in marked contrast to the monotonic increase in synchronization with increasing 


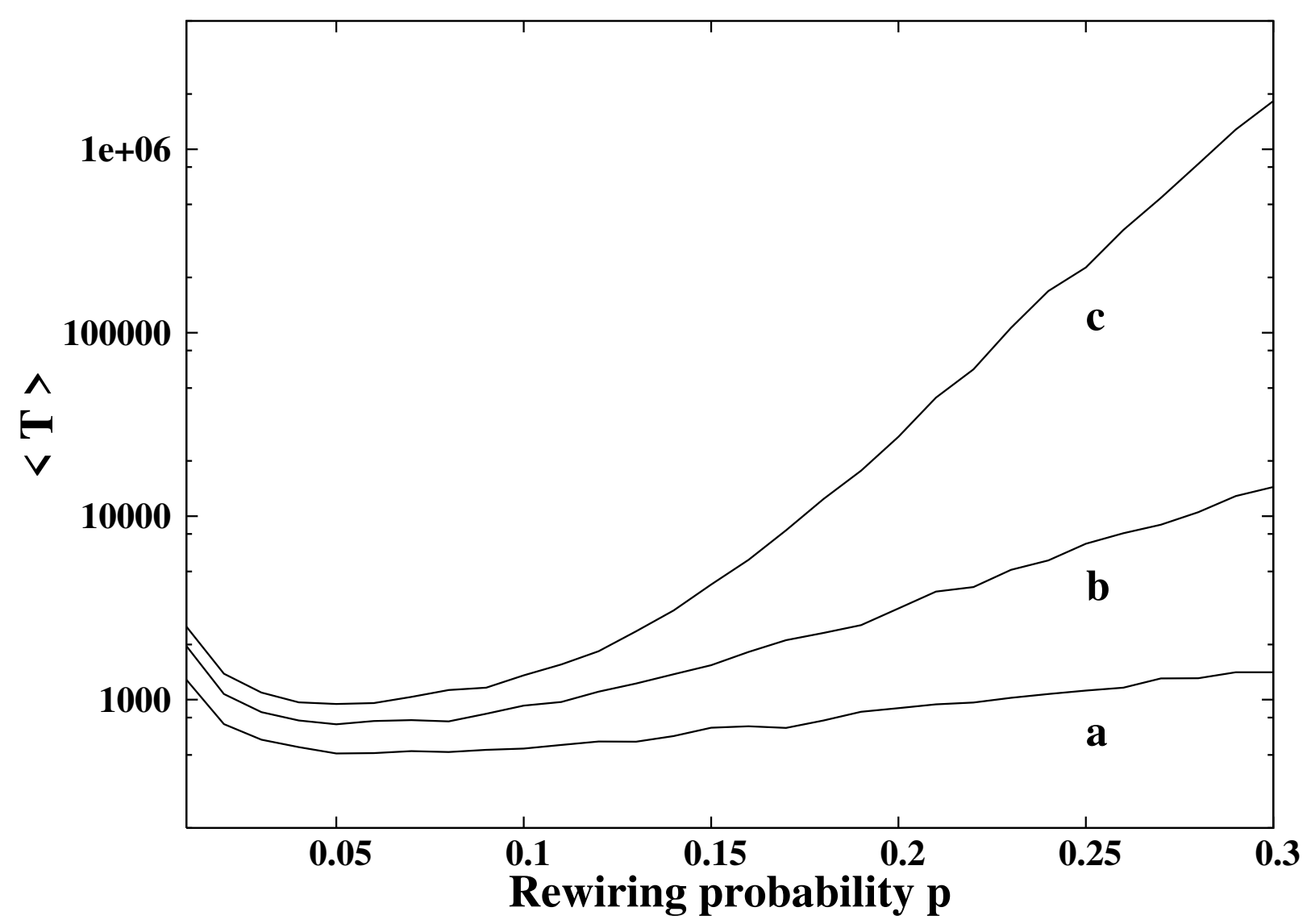

FIG. 5: Average time required to reach synchronization $\langle T\rangle$ vs rewiring probabilty $p$, for coupling strength $\epsilon=0.19$ for system sizes $N=25,50,100$. The synchronized state is a global attractor for the system for the values of $p$ displayed here.

randomness in connectivity, observed in the strong coupling regime of the same system.

[1] R. Albert and A.-L. Barabasi, Rev. of Mod. Phys. 74, 47 (2002), A.-L. Barabasi, 'Linked: The New Science of Networks' (Massachusetts: Persus Publishing, 2002); T. Gross and B. Blasius, J. of Royal Soc. - Interface 5259 (2008).

[2] D. J. Watts and S. H. Strogatz, Nature, 393440 (1998).

[3] S. A. Pandit and R. E. Amritkar, Phys. Rev. E 63041104 (2001), M. E. J. Newman and D.J. Watts, Phys. Rev. E 607332 (1999); A. Barrat and M. Weigt, Eur. Phy. J. B 13547 (2000); S.C. Manrubia, J. Delgado, and B. Luque, Europhys. Lett. [53], 693 (2001). 


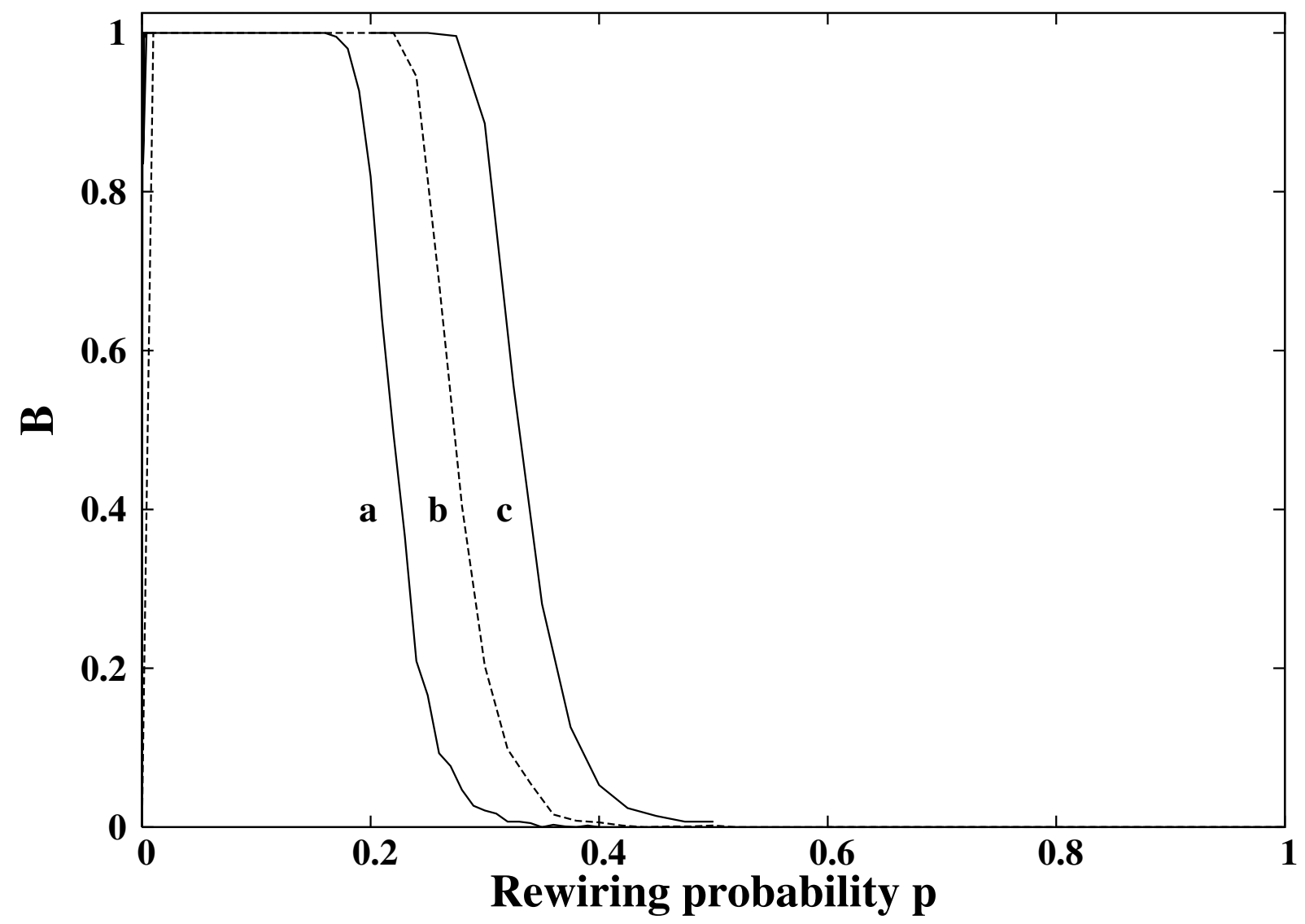

FIG. 6: Size of the basin of attraction of the synchronized state $B$ vs rewiring probability $p$, for coupling strength $\epsilon$ for a system of size 100 for maximum allowed transience equal to (a) $5 \times 10^{4}$ (b) $5 \times 10^{5}$ (c) $5 \times 10^{6}$.

[4] K. Kaneko, Theory and Applications of Coupled Map Lattices, Wiley, New York (1993)

[5] P. M. Gade and C.-K. Hu, Phys. Rev. E 626409 (2000); Z. Gao, B. Hu and G. Hu, Phys. Rev. E 65016209 (2001); M. Barahona and L. Pecora, Phys. Rev. Lett., 89, 054101 (2002); J. Jost and M. P. Joy, Phys. Rev. E 65 016201(2002); M. G. Cosenza and K. Tucci, Phys. Rev. E, bf 650326223 (2002); P. R. A. Campos, V. M. de Oliveira and F. G. Brady Moreia, Phys. Rev. E 67026104 (2003); P.M. Gade and S. Sinha, Int. J. of Bif. and Chaos, 162767 (2006); M.P.K. Jampa et al, Phys. Rev. E, 75026215 (2007); F. Radicchi and H. Meyer-Ortmanns, Phys. Rev. E 74026203 (2006); T. Gross, C.J.D'Lima and B. Blasius, Phys. Rev. Letts. 96208701 (2006); S. Rajesh et al, Phys. Rev. E, 75011906 (2007).

[6] S. Sinha, Phys. Rev. E, 66, 016209 (2002) 